\title{
The Problem of Fuzzy Maximal Flow- Fuzzy Minimal Cut
}

\author{
Rostin MABELA MAKENGO MATENDO ${ }^{1}$, Fernand MAMANYA TAPASA ${ }^{2 *}$, Jean-Pierre MUKEBA \\ KANYINDA ${ }^{3}$ \\ ${ }^{1}$ Department of Maths and Computer Science, Faculty of Science, University of Kinshasa, D.R. Congo \\ ${ }^{2}$ Department of Maths and Computer Science, Faculty of Science, National Pedagogic University, Kinshasa, \\ D.R. Congo \\ ${ }^{3}$ Department of Maths and Computer Science, Teacher Training College of Mbuji-Mayi, D.R. Congo
}

*Corresponding Author: Fernand MAMANYA TAPASA, Department of Maths and Computer Science, Faculty of Science, National Pedagogic University, Kinshasa, D.R. Congo

\begin{abstract}
In this paper, we state and we prove the fuzzy version of the maximal flow-minimal cut theorem, which was stated in the past in crisp environment by Ford and Fulkerson. To illustrate this result, we use triangular fuzzy numbers under alpha-cut approach.
\end{abstract}

Keywords: Maximal fuzzy flow, minimal fuzzy cut, alpha cut, fuzzy triangular numbers.

\section{INTRODUCTION}

The maximal flow-minimal cut problem is much studied in the literature (cfr [5], [4], [12]), due to its numerous applications, namely in the conception and the exploitation of the telecommunication networks, in the exploitation of the road transportation networks, in the water adduction systems, in the electric networks, in the computer networks, etc. It's a problem of transportation where a product is conveyed from the source of this product to its destination via a network, for instance a road network.

Each road has a transportation capacity. The network which connects the origin to the destination can be modeled by the Graph Theory as a transportation network. One is interested then, to the link (relation) between the maximal flow quantity which circulates in the network and the capacity of edges which transports this maximal flow.

Apart from the introduction and the conclusion, this paper is structured as follows:

The second section is devoted to the fuzzy arithmetic, the third section presents the literature review, the fourth and fifth sections give a glance on the classical maximal flow and minimal cut problem, respectively.

In the remaining part of this paper, we deal with the fuzzy flow problem, the fuzzy cut problem and the maximal fuzzy flow-minimal fuzzy cut theorem.

\section{FUZZY ARITHMETIC BASEd ON AlPha-CUTS AND ARITHMETIC INTERVALS.}

Proposition 2.1 (Cfr Ritha \& Menon [13]).

Let $\widetilde{Q}$ and $\tilde{Q}^{\prime}$ be two fuzzy numbers with respective $\alpha-$ cuts $\widetilde{Q}_{\alpha}$ and $\tilde{Q}_{\alpha}^{\prime}$, with $\alpha \in[0,1]$.

The operations,+- , x and $\div$ are defined on $\tilde{Q}$ and $\tilde{Q}^{\prime}$ via their $\alpha$-cuts as follows :

$\left(\tilde{Q}+\widetilde{Q}^{\prime}\right)_{\alpha}=\tilde{Q}_{\alpha}+\widetilde{Q}_{\alpha}^{\prime}$

$\left(\tilde{Q}-\widetilde{Q}^{\prime}\right)_{\alpha}=\tilde{Q}_{\alpha}-\widetilde{Q}_{\alpha}^{\prime}$

$\left(\tilde{Q} \times \widetilde{Q}^{\prime}\right)_{\alpha}=\tilde{Q}_{\alpha} \times \widetilde{Q}_{\alpha}^{\prime}$

$\left(\tilde{Q} \div \widetilde{Q}^{\prime}\right)_{\alpha}=\tilde{Q}_{\alpha} \div \widetilde{Q}_{\alpha}^{\prime}$ 
The four equalities above are summarized in the general proposition stated by Hanss [3], for a given fuzzy arithmetic operations as follows:

Let $\widetilde{Q}$ and $\widetilde{Q}^{\prime}$ be two fuzzy numbers with respective $\alpha-$ cuts $\widetilde{Q}_{\alpha}$ and $\tilde{Q}_{\alpha}^{\prime}$, with $\alpha \in[0,1]$. We have:

$\left(\tilde{Q} \otimes \widetilde{Q}^{\prime}\right)_{\alpha}=\widetilde{Q}_{\alpha} * \widetilde{Q}_{\alpha}^{\prime}$

Where * is an arithmetic operation defined in the family of closed intervals of $\mathbb{R}$ by :

$\forall\left[a_{1}, b_{1}\right],\left[a_{2}, b_{2}\right] \in \mathbb{R}$,

$\left[a_{1}, b_{1}\right] *\left[a_{2}, b_{2}\right]=[\min G, \max G]$

Where,

$G=\left\{a_{1} * a_{2}, a_{1} * b_{2}, b_{1} * a_{2}, b_{1} * b_{2}\right\}$

Mukeba [11] gives the following schema:

$\left.1^{\circ}\right)$ The defuzzification : write the $\alpha$-cuts of the concerned fuzzy numbers in the form of classical intervals. In particular, if $\widetilde{Q}=(a, b, c)$ is a triangular fuzzy number, then :

$\tilde{Q}_{\alpha}=[a+(b-a) \alpha, c+(b-c) \alpha], \quad \forall \alpha \in[0,1]$

$\left.2^{\circ}\right)$ The ordinary calculations : application of relation (6).

$\left.3^{\circ}\right)$ The fuzzification : fuzzify the result obtained above by applying the following relation:

$\eta_{\tilde{Q}}(x)=\sup _{\alpha \in[0,1]} \min \left\{\alpha, \eta_{\tilde{Q}_{\alpha}}(x)\right\}$

Where

$\eta_{\tilde{Q}_{\alpha}}(x)=\left\{\begin{array}{lr}1 & \text { si } x \in \tilde{Q}_{\alpha} \\ 0 & \text { otherwise }\end{array}\right.$

Where $\eta_{\tilde{Q}}$ is the membership function of the fuzzy number $\tilde{Q}$.

Thus, $\forall\left[a_{1}, b_{1}\right],\left[a_{2}, b_{2}\right] \subset \mathbb{R}$ :

$\left[a_{1}, b_{1}\right] *\left[a_{2}, b_{2}\right]=\left\{a * b \mid a_{1} \leq a \leq b_{1}\right.$ et $\left.a_{2} \leq b \leq b_{2}\right\}$

$\left[a_{1}, b_{1}\right]+\left[a_{2}, b_{2}\right]=\left[a_{1}+a_{2}, b_{1}+b_{2}\right]$

$\left[a_{1}, b_{1}\right]-\left[a_{2}, b_{2}\right]=\left[a_{1}-b_{2}, b_{1}-a_{2}\right]$

$\left[a_{1}, b_{1}\right] \times\left[a_{2}, b_{2}\right]=\left[\min \left(a_{1} a_{2}, a_{1} b_{2}, b_{1} a_{2}, b_{1} b_{2}\right), \max \left(a_{1} a_{2}, a_{1} b_{2}, b_{1} a_{2}, b_{1} b_{2}\right)\right]$

$\frac{\left[a_{1}, b_{1}\right]}{\left[a_{2}, b_{2}\right]}=\left[\min \left(\frac{a_{1}}{a_{2}}, \frac{a_{1}}{b_{2}}, \frac{b_{1}}{a_{2}}, \frac{b_{1}}{b_{2}}\right), \max \left(\frac{a_{1}}{a_{2}}, \frac{a_{1}}{b_{2}}, \frac{b_{1}}{a_{2}}, \frac{b_{1}}{b_{2}}\right)\right]$

The multiplication and the division are simplified if the information on the signs of the bounds is available.

\section{LITERATURE REVIEW}

The problem of flow has been studied for the first time by Harris and Ross in 1954 ([2] and [6]). To solve it, Ford and Fulkerson set up the well-known algorithm based on the flow increase along the edges about the end of 1956 [2]. Ever since, it constitutes an important research area due to its numerous applications evoked above [6]. This famous algorithm has been enriched by the contribution of many variants. Rozemberg [14] has investigated the "minimum cost flow problem". He has introduced the fuzzy flow problem and proposed a dynamical programming based resolution method [12]. Liu and Kao have studied the flow problem in a transportation network using fuzzy edges.

Bagherian has proposed a fuzzy residual network approach to solve the minimum cost flow problem with fuzzy parameters [1]. In the same period, other research works on the minimum cost fuzzy flow problem where published by Ghatee, Grupta and Pal, Okada and Gent []. Karzanov has set up a flow increasing based method to determine the maximal flow [5]. Nawathe \& Kao has focused their research on the study of the maximal flow distribution in a transportation network by exploiting the Karzonov's method [6]. Dinic has investigated the problem of flow distribution in a transportation network with 
fuzzy capacities, using the comparison methods []. Kumar and Kaur [6] have solved the maximal fuzzy flow problem by using the linear programming, but they concluded that it was not possible to obtain the optimal solution because of the heaviness of the fuzzy tasks.

Those various researches on the flow enabled to improve the understanding and the resolution methods of the flow and cut problem in a deterministic environment.

\section{THE CLASSICAL FLOW PROBLEM}

\subsection{Definition of the problem}

Let's consider a network $(P, K)$ on the set of communications where a function of edges capacities $c(i, j) ; k_{i j}=(i, j) \in K$

Let's select two points of the network $p_{1}$ and $p_{n}$, where $p_{1}$ is called "origin" and $p_{n}$ the "destination".

Let $E_{i}$, the set of communications of the network outgoing from $p_{i}$, and $E_{i}^{\prime}$ the set of communications entering into $p_{i}$.

A function $x$ defined on the set $K$ by:

$x(i, j)=x_{i j}$, satisfying the following conditions ([9], [4]):

$\sum_{k_{i j} \in E_{i}} x_{i j}-\sum_{k_{i j} \in E_{i}^{\prime}} x_{i j}=0 \quad \forall i \neq 1, n$

$x_{1}=\sum_{k_{i j} \in E_{i}^{\prime}} x_{i j}-\sum_{k_{i j} \in E_{1}} x_{i j} \geq 0$

$\forall k_{i j} \in K ; 0 \leq x(i, j) \leq c(i, j)$

is called "network flow" compatible with the function $c(i, j)$;

$k_{i j} \in K$; and directed from $p_{1}$ and $p_{n}$.

A flow represents a flux of material routing from a source "s" to a destination " $t$ ".

The equation (7) indicates that the flow quantity on an edge must be less or equal to the edge capacity. Furthermore, it's not possible to store or to produce material on intermediate nodes. This property is translated by the equation (15). The relation (16) translates the principle of the flow conservation.

\subsection{The Maximal flow problem}

Given the edges capacities in a transportation network, the maximal flow problem consists to find the maximal quantity of flow which can be routed from the source to the destination. Ford and Fulkerson's algorithm ([9], [4]) is well known to solve this problem.

\section{The Classical Minimum Cut Problem in a Transportation Network}

Let $s$ and $t$ be two vertices of a network $R=(P, K)$.

- $\quad$ A $(s, t)$ cut is a set $C$ of edges disconnecting $s$ and $t:$ in the partial graph $(P, K \backslash C)$, there doesn't exist any directed path from $s$ and $t$.

- $\quad$ A $(s, t)$ cut is also defined by a partition $C=S \cup T$ of vertices such that $s \in S$ and $t \in T$.

Then the edges $(x, y)$ of a cut are those one having their origin $x$ in $S$ and their destination $y$ in $T$.

The capacity of a cut is the sum of capacities of the edges which compose this cut.

The problem of "minimum cut" consists to find a cut $C_{\min }$ between $s$ and $t$ of a minimum capacity. ([9], [4]).

A cut is an obliged passage for any flow. In fact, any flow transiting from and $s$ to $t$ must necessarily "borrow" the edges of the cut (any path from $s$ to $t$ includes at least one by definition). The value of the flow can be redefined as the difference between the outgoing flow and the entering one. 


\section{Theorem $5.1([5] \&[12])$}

In a transportation network carrying a flow, the following assertions are equivalent:

(1) The flow is maximal

(2) There exists no increasing path from the source $s$ to the destination $t$.

(3) There exists a maximal flow $x_{\max }$ less or equal than the capacity of the cut.

\section{Theorem 5.2 ([2])}

The maximal value of a flow from a source $s$ to a destination $t$ in a network is equal to the minimal capacity of a (s,t)-cut.

So, we have :

$x_{\max }=C_{\min }$

\section{The Problem of FuZzy Flow}

Let $(P, K)$ be a transportation network where a capacity function $\widetilde{C}$ is defined. To edge $k_{i j}=(i, j) \in$ $K$, a fuzzy positive number $\tilde{C}_{i j}$ is associated. Let's select two vertices of the graph denoted by $p_{1}$ and $p_{n}$, where $p_{1}$ is the entrance and $p_{n}$ the exit.

Let $E_{i}$, the set of communications of the network outgoing from $p_{i}$, and $E_{i}^{\prime}$ the set of communications entering into $p_{i}$.

As all the edges are positively valuated by fuzzy numbers, this graph is a fuzzy transportation network. We denote it by $(P, K, \tilde{C})$, where $\tilde{C}$ is the set of fuzzy capacities.

A fuzzy function $\tilde{x}$ defined on the set $\mathrm{K}$, associating to each edge $(i, j) \in K$, a fuzzy number $\tilde{x}_{i j}$ and satisfying the following conditions :

$$
\begin{array}{ll}
\sum_{k_{i j} \in E_{i}} \tilde{x}(i, j)-\sum_{k_{i j} \in E_{i}^{\prime}} \tilde{x}(j, i)=0 & \forall i \neq 1, n \\
\tilde{x}_{1}=\sum_{k_{i j} \in E_{i}^{\prime}} \tilde{x}(j, i)-\sum_{k_{i j} \in E_{1}} \tilde{x}(i, j) \geq 0 & \\
\forall k_{i j} \in K ; 0 \leq \tilde{x}(i, j) \leq \tilde{c}(i, j) &
\end{array}
$$

Is called a fuzzy flow of the network $(P, K)$ consistent with the function $\widetilde{C}$ and directed from $p_{1}$ and $p_{n}$. The sense of the so introduced terms can be interpreted in an analogous way as the deterministic flows.

\section{Remark 6.1}

The inequality " $\leq$ " in equation (20) is different from the one defined in Mukeba [10]. To define it, we are guided by the fuzzy order defined used by Liou and Wang [8], Kumar and Kaur [7], as follows :

$\tilde{A} \leq \tilde{B} \Leftrightarrow a+2 b+c \leq d+2 e+f$

\section{Problem of Maximum Fuzzy Flow}

\subsection{Definitions}

The details in this section are drawn from F. Mamanya and R. Mabela [2].

- $\quad$ A fuzzy directed edge $(i, j)$ is saturated if the modal value of this capacity called "fuzzy number kernel" is equal to the modal value of the current flow.

For the particular case of the fuzzy triangular numbers : if the capacity of the fuzzy edge is

$\tilde{c}_{i j}=(a, b, c)$ and the value of the flow is $\tilde{x}_{i j}=(d, e, f)$, with

$b=e$, the edge is saturated.

A fuzzy path is saturated if it contains at least one saturated edge. 
- $\quad$ A fuzzy flow is complete if all the paths from source to the destination are saturated.

To saturate a fuzzy edge or a fuzzy path, one adds to this edge or this path the residual flow.

The residual fuzzy flow on an edge $k(i, j)$ denoted $\tilde{x}_{r}(i, j)$ is equal to the difference between the fuzzy capacity of this edge and its fuzzy flow:

$\tilde{x}_{r}(i, j)=\tilde{c}(i, j)-\tilde{x}(i, j)$

The fuzzy residual flow of a path $\Phi$ is the minimum of the fuzzy residual flows of the fuzzy edges which compose this fuzzy path:

$\tilde{x}_{r}(\Phi)=\min _{(i, j) \in \Phi}\{\tilde{c}(i, j)-\tilde{x}(i, j)\}$

The fuzzy residual graph is the one which contains the same vertices as the initial graph but no saturated fuzzy edge. It contains two kinds of edges :

The fuzzy direct edges $(i, j)$ whose fuzzy flow is given by $: \tilde{c}(i, j)-\tilde{x}(i, j)$

The reverse fuzzy edges $(i, j)$ whose fuzzy flow is equal to the modal value of the capacity of the fuzzy edge (not null).

- $\quad$ The increase of the fuzzy flow consists to add the minimum value of deviations between the current fuzzy flow and the capacities of all the fuzzy flows of the path.

\subsection{Statement of the Maximal Fuzzy Flow Problem.}

Given the capacities of the edges of a fuzzy transportation network, determine the maximal flow quantity which circulate from the source to the destination.

\section{Fuzzy Minimal CuT}

The problem of the fuzzy minimal cut consists in finding a cut $C_{\text {min }}$ from $s$ to $t$ having a minimal capacity. A cut is an obliged passage for a fuzzy flow. In fact, a fuzzy flow in transit from $s$ to $t$ should "borrow" the edges of a cut (any path from $s$ to $t$ has at least one, by definition). The value of the fuzzy flow can be defined as the difference between the fuzzy flow outgoing from the cut and the entering fuzzy flow.

\section{The MaXimal Fuzzy Flow Theorem-Minimal Fuzzy Cut}

The problem of maximal fuzzy flow theorem-minimal fuzzy cut has not been addressed yet in the scientific literature. We give, in the remainder of this paper, a first proposal. In a transportation network carrying a fuzzy flow $\tilde{x}$ evaluated by triangular fuzzy numbers, the modal value of the maximal fuzzy flow is equal to the value of minimal fuzzy cut:

$\tilde{x}_{\max }=\tilde{c}_{\min }$

Proof:

Let's prove that the three following assertions are equivalent:

(i) The fuzzy flow is maximal

(ii) There doesn't exist any increasing fuzzy path between $s$ and $t$ for the fuzzy flow $\tilde{x}$

(iii) There exists a fuzzy flow $\widetilde{x}$ whose modal value is equal to the cut capacity $\tilde{c}$

To set the equivalence between those three assertions, we'll establish the sequence of the following implications:

(i) $\Rightarrow$ (ii)

If there may exist an increasing residual fuzzy path $\tilde{X}_{r}$, then $\tilde{X}+\tilde{X}_{r}$ would be a fuzzy flow. That contradicts the fact that $\tilde{X}$ is a maximal fuzzy flow.

$(i i) \Rightarrow(i i i)$

Let's prove that the value of maximal fuzzy flow is equal to the value of minimal fuzzy cut.

From the following relation : 
$\tilde{X}=\tilde{X}^{+}(S)-\tilde{X}^{-}(S)$

We see that all the fuzzy edges of the cut outgoing from $S^{+}$are saturated by the fuzzy flow $\tilde{X}$ and no flow is in transit on all the fuzzy edges entering into $S^{-}$. Let's consider a fuzzy edge $(x, y)$, where $x \in$ $S$ and $y \in T$ and let's assume that $(x, y)$ is not saturated. Then, there exists a fuzzy path from $s$ to $x$. Extending it to the edge $y$, we obtain then a path from $s$ to $y$. This contradicts the fact that $y \in T$.

Let's consider an edge $(y, x)$, where $y \in T$ and $x \in S$. Let's assume by absurd that the fuzzy flow $\tilde{X}$ is not null on this edge, then there exists, in the residual graph, a reverse edge $(y, x)$ with a non-null capacity. Contradicting the fact $y \notin S$.

$\tilde{X}=\tilde{X}^{+}(S)-\tilde{X}^{-}(S)=\sum_{x \in S} \sum_{y \in T} \tilde{c}(x, y)-0=\tilde{C}$

(iii) $\Rightarrow($ i). As mentioned above in the subsection 7.1, the edge whose modal value of the fuzzy flow is equal to the capacity is saturated. The path from $s$ to $t$ leads to the conclusion that the flot $\tilde{X}$ is maximal.

\section{NUMERICAL EXAMPLE}

Let's determine the maximal fuzzy flow and the minimal fuzzy cut on the following transportation network:

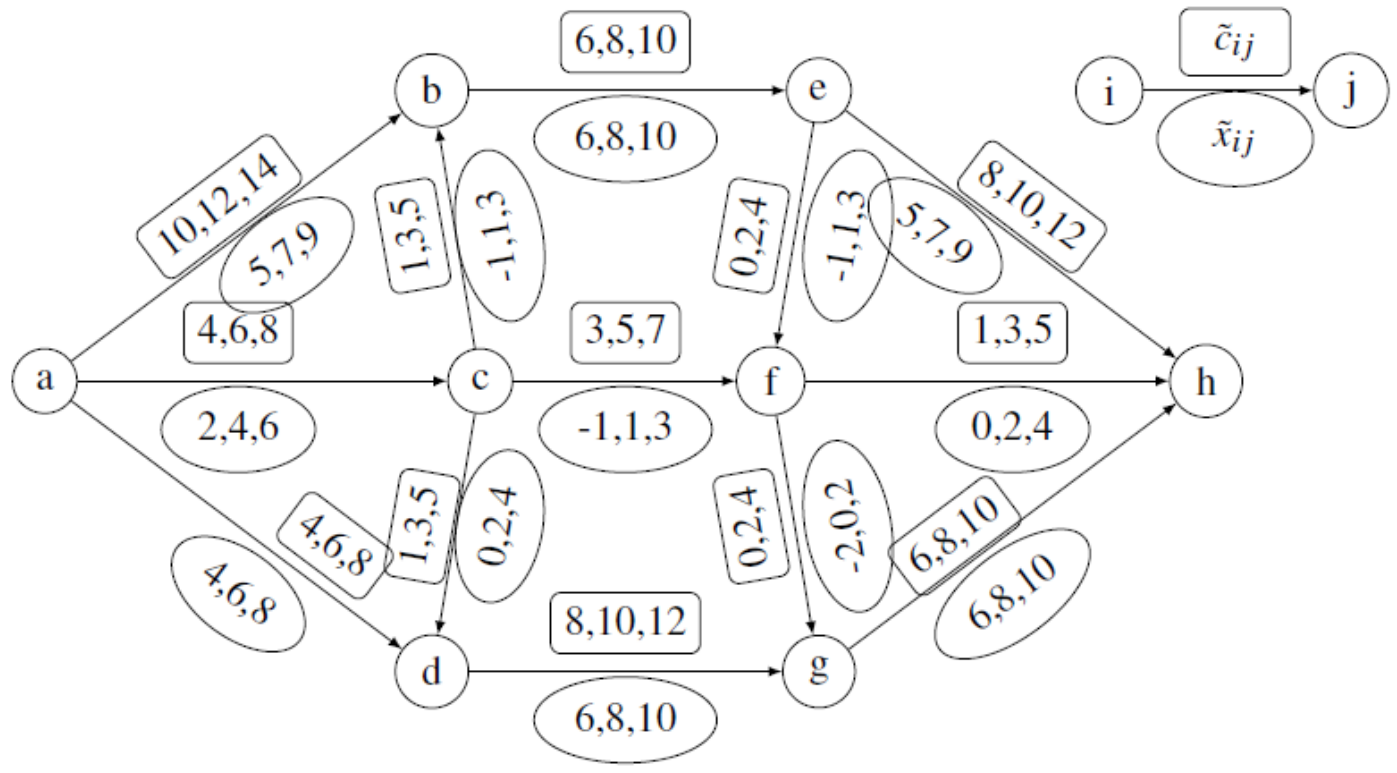

Fig1. A transportation network able to carry fuzzy flows.

The value of fuzzy entry flow $=(11,17,23)=$ the value of fuzzy outgoing flow. On each node of the network, the sum of entering modal values= the sum of outgoing modal values. The principle of fuzzy flow conservation is satisfied. Then, the network under investigation is able to let the fuzzy flow pass.

\section{Step 1: Searching a complete fuzzy flow}

Searching the fuzzy flows from to $h$ : a-b-e-h, a-b-e-f-h, a-b-e-f-g-h, a-c-f-h, a-c-d-g-h, a-c-dg-h, a-c-f-g-h, a-d-g-h : 7 paths.

- $\quad$ Determination of non-saturated paths : only one : a-c-f-h

- $\quad$ The entering flow is not complete

- $\quad$ Saturation of the path : a-c-f-h

One computes the residual fuzzy flow on the non-saturated path.

$\tilde{x}_{r}(a-c-f-h)=\min \{(4,6,8)-(2,4,6),(3,5,7)-(-1,1,3),(1,3,5)-(0,2,4)\}$

The calculation is done by the $\alpha-c u t$ approach and intervals proposed by [11], and the max-min approach to compare the fuzzy numbers.

$\tilde{x}_{r}(a-c-f-h)=\min \{(-2,2,6),(0,4,8),(-3,1,5)=\{(-3,1,5)\}\}$ 
We add to all edges path a-c-f-h to all on every edges of the path a-c-f-h, the residual fuzzy flow. Then, the graph becomes:

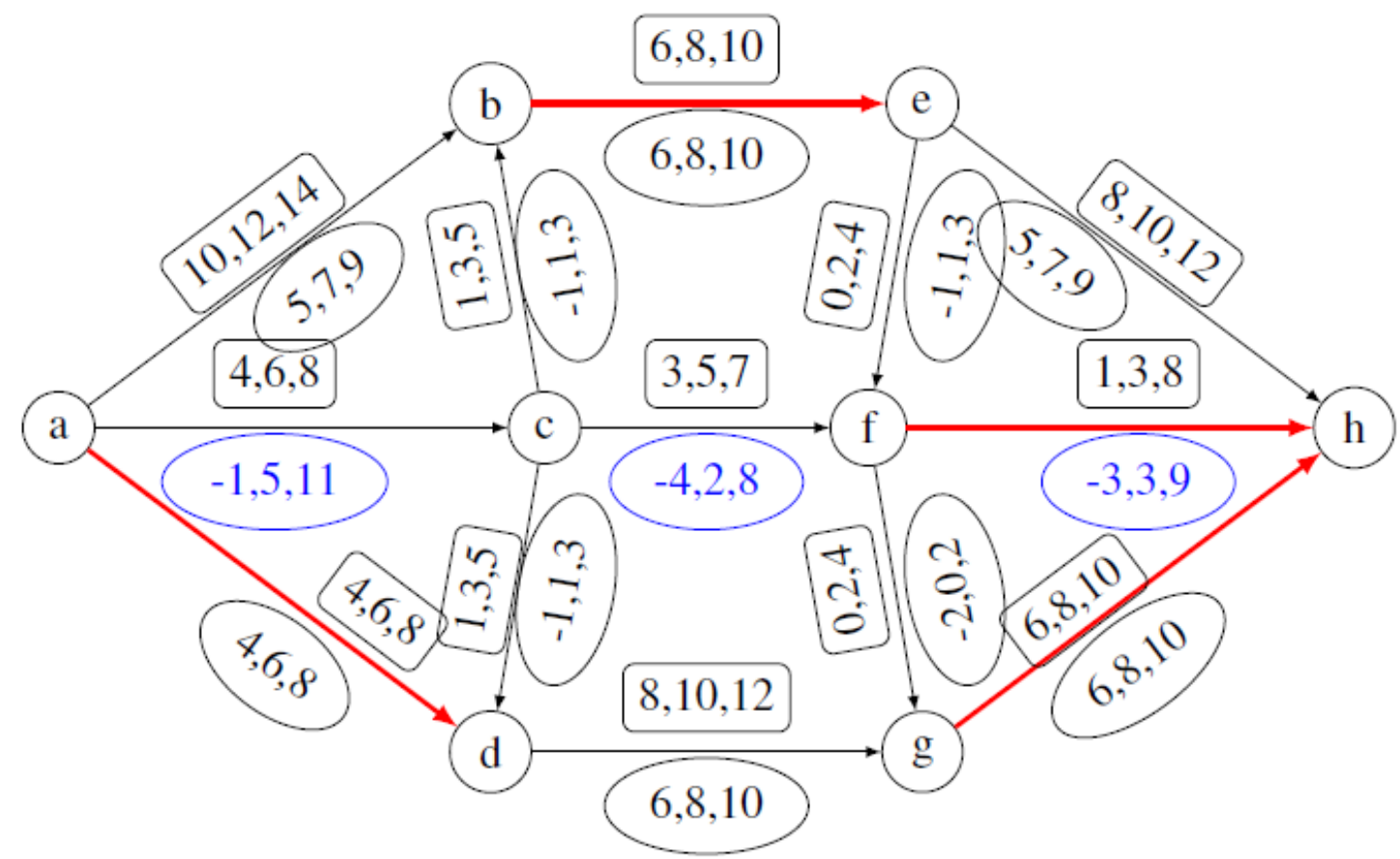

Fig2. Network expressing the complete fuzzy flow.

One notices that the value of entering fuzzy flow $=(8,18,28)=$ the value of outgoing fuzzy flow. We notice that all the paths from $a$ to $h$ are saturated. So, the fuzzy flow is complete. Its value is $(8,18,28)$. This flow is not necessarily maximal.

Step 2: Constructing the residual fuzzy graph : increasing path and marking.

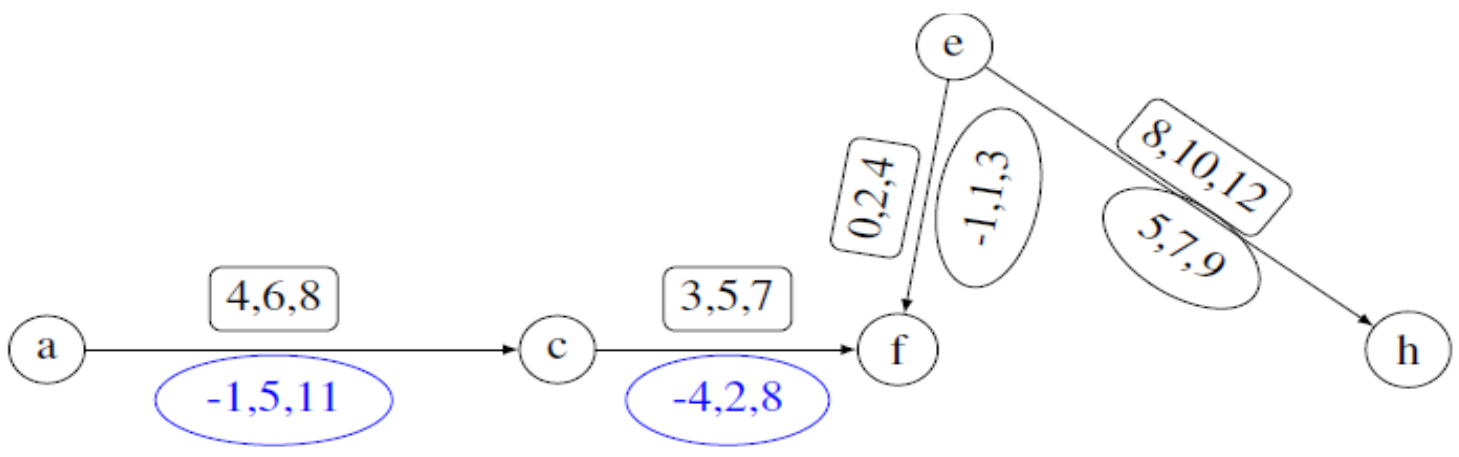

Fig3. Path which may be increased

By the procedure of marking, we search the increasing path from $a$ to $h$.

One marks the node $a$ by writing $a+, c$ by c+, $f$ by $f+$, e+ and finally $h+$. The fuzzy obtained is complete but it's not maximal.

\section{Step 3: Determining the maximal fuzzy flow}

It's concerning the improvement of the fuzzy flow on the increasing chain:

$a-c-f-e-h$.

One calculates the fuzzy residual flow on the chain $a-c-f-e-h$.

$$
\begin{gathered}
\tilde{x}_{r}=\min \{(4,6,8)-(-1,5,11),(3,5,7)-(-4,2,8),(0,2,4)-(-1,1,3),(8,10,12)-(5,7,9)\} \\
\tilde{x}_{r}=\min \{(-7,1,9),(-5,3,11),(-3,1,5),(-1,3,7)\}=\{\{(-3,1,5)\}
\end{gathered}
$$

Nota : To increase the fuzzy flow on the reverse fuzzy edge $f$-e means to reduce the flow on the edge $f$ $e$. Then, the path becomes: 


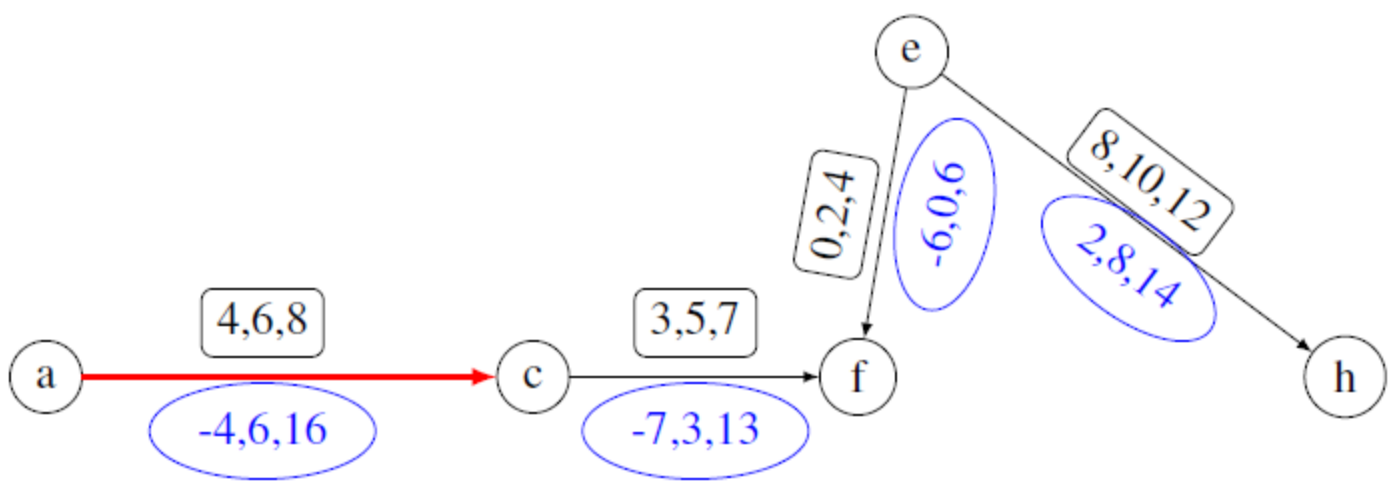

Fig4. Increasing path from a to $h$.

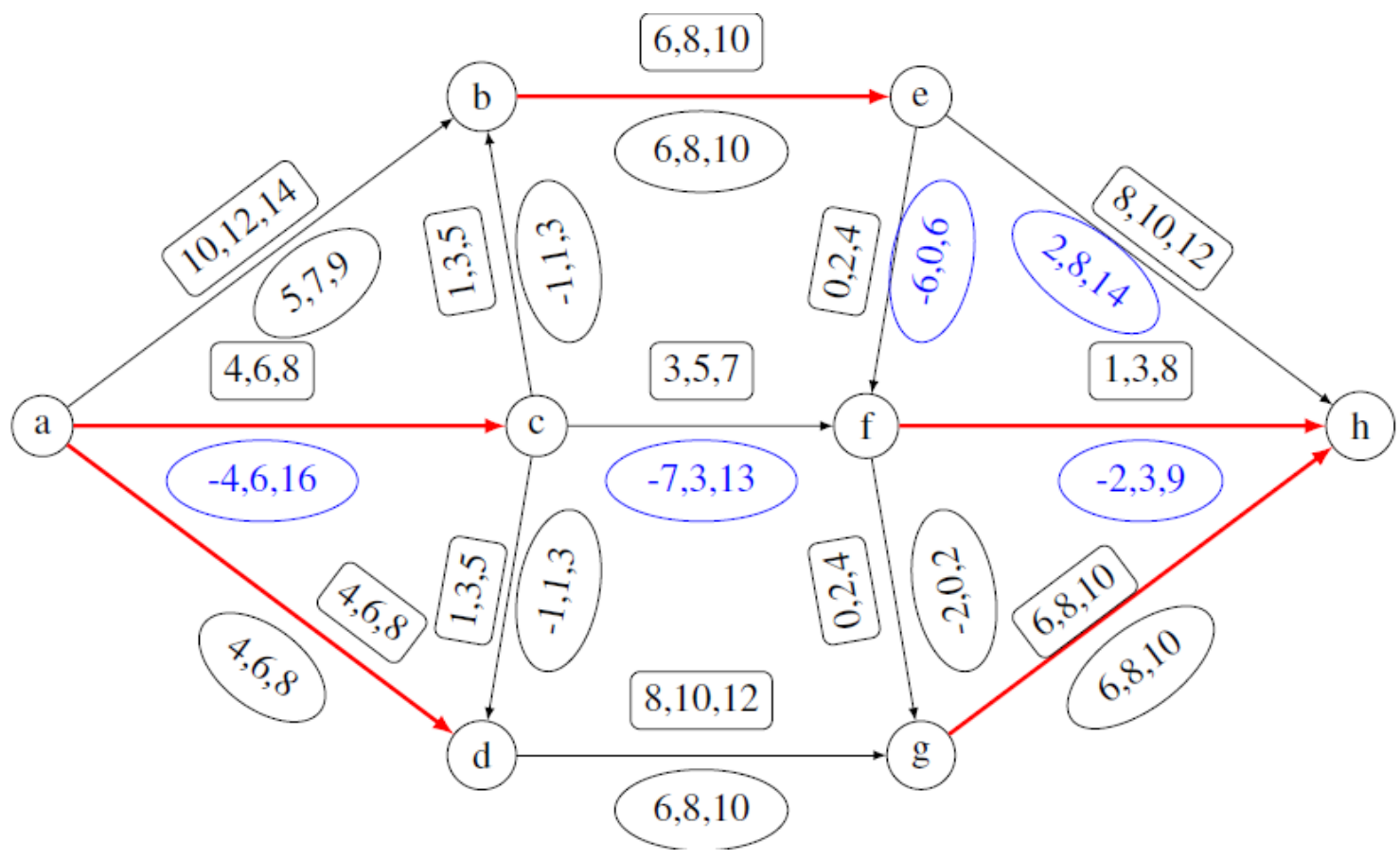

Fig5. Network carrying the maximal fuzzy flow.

The value of entering fuzzy flow is $=(5,19,33)=$ the value of fuzzy outgoing flow. This value is also equal to the value of the minimal cut passing by $(g, h),(f, h),(e, f)$ and $(b, e)$. At each node, the sum of central values of entering fuzzy flows is equal to the sum of central values of outgoing fuzzy flows. The principle of conservation is satisfied. The maximal flow is then $(5,19,33)$.

Interpretation: The flow circulating through the network can vary between 5 and 35 . Its minimal value can't be less than 5, while its maximal value can't exceed 33. The modal value 19 is the most frequent one. The maximal fuzzy flow is about $(5,19,33)$.(cfr Fig.5).

\section{CONCLUSION}

In this paper, we have stated and proved the theorem of maximal flow - minimal cut in a fuzzy environment. We have used the fuzzy triangular numbers to illustrate this result. In a transportation network carrying a fuzzy flow, the value of maximal fuzzy flow is equal to value of the minimal fuzzy cut. In our forthcoming researches, we'll investigate on the theorem of maximal fuzzy multiflow minimal fuzzy multicut.

\section{REFERENCES}

[1] M. Bagherian. A Fuzzy Residual Network Approach to Minimum Cost Flow with Fuzzy Parameters. ARPN Journal of Science and Technology, 2(10), 2012.

[2] L.R. Ford and D.R. Fulkerson, Flows in Networks. Princeton University Press, New York, 1962.

[3] M. Hanss. Applied Fuzzy Arithmetic, An Introduction with Engineering Applications. Springer Verlag. Berlin Heidelberg, 2005. 
[4] R. Howat, G. Kos and M. Secrovic. Tekenicki Vjesnik. Sov. Math, Dokdy, 22 (2) : 475-486, 2015.

[5] Karzonov, Determining the maximum flow in a network by the method of preflow. Sov. Math. Dokldy, 15(3): 43-47, 1974.

[6] A. Kumar and M. Kaur. A New Algorithm for Solving Network Flow Problem with Fuzzy Lengths. Turkish Journal of Fuzzy Systems 2(1):

a. $1-13,2013$.

[7] A. Kumar and M. Kaur. A New Algorithm for Solving Network Flow Problem with Fuzzy Lengths. TJFS. Turkish Journal of Fuzzy Systems (eISSN : 1309-1190), 2(1), 2011.

[8] T.S. Liou and M. J. Wang. Ranking Fuzzy Numbers with Integral Value. Fuzzy Sets and Systems, 50: 247-255, 1992.

[9] F. Mamanya and R. Mabela. Un Nouvel Algorithme pour la Détermination de Flot Maximal. International Journal of Innovation and Applied Studies, ISSN 2028-9324, 21(3) : 492-498, 2017.

[10] J.P. Mukeba. Analysis of Fuzzy Queue Characteristics by Flexible Alpha-Cuts Method. Journal of Fuzzy Sets Valued Analysis, 2017(1) : 1-11, 06 March 2017.

[11] J.P. Mukeba. Chaînes de Markov Floues et Evaluation des Paramètres de Performance d'un Réseau de Files d'Attente Floues à Forme Produit. PhD Thesis, Université Pédagogique Nationale de Kinshasa, R.D. Congo, 26 Novembre 2016.

[12] R. Renfrey Potts. Flows in Transportation Networks. Academic Press, New-York and London, 1972.

[13] W. Ritha and S.B. Menon. Fuzzy N. Policy Queues with Infinite Capacity. Journal of Physical Sciences, 15: 73-82, December 2011.

[14] I. Rozenberg and T. Starostina. Minimal Cost Flow Problem in a Graph with Flow Intensification and Fuzzy Parameters. In 13 Zittau East West Fuzzy Colloquium, Hochschule Zitau Goerlitz, Zittau, pages 176-184, 2006.

Citation: Fernand MAMANYA TAPASA,et.al., The Problem of Fuzzy Maximal Flow- Fuzzy Minimal Cut, International Journal of Scientific and Innovative Mathematical Research (IJSIMR), vol. 8, no. 4, pp. 9-17, 2020. Available : DOI: http://dx.doi.org/10.20431/2347-3142.0803004

Copyright: ( 2020 Authors. This is an open-access article distributed under the terms of the Creative Commons Attribution License, which permits unrestricted use, distribution, and reproduction in any medium, provided the original author and source are credited. 